\title{
Comunicación
}

\section{Ocurrencia de Prostenorchis elegans en un mono tocón (Plecturocebus oenanthe) reinsertado en el bosque del Área de Cordillera Escalera, Región San Martín}

\author{
Occurrence of Prostenorchis elegans in the San Martin titi monkey \\ (Plecturocebus oenanthe) reinserted in the tropical forest of the \\ national park Coordillera Cerro Escalera, San Martin Region
}

\author{
Alicia López F. ${ }^{1,4}$, Víctor Puicón N. ${ }^{2,5}$, Fredy Fabián D. ${ }^{1}$, Isaí Bustamante Q. ${ }^{1}$, \\ Rodrigo García R. ${ }^{1}$, Daniel Vecco G. ${ }^{3}$
}

\section{Resumen}

Se reporta el caso de un mono tocón adulto, macho (Plecturocebus oenanthe) parasitado con Prosthenorchis elegans, fallecido a fines de diciembre de 2019, en un centro de custodia de fauna silvestre ubicado a orillas de la Quebrada Shilcayo, puerta de entrada del Área de Conservación Regional «Cordillera Escalera», San Martín, Perú. El animal, en semi-cautividad, no presentó signos clínicos, pero se desplomó desde una altura de $5 \mathrm{~m}$. A la necropsia, se evidenció exudado abdominal, perforación a nivel del intestino delgado, congestión de vasos sanguíneos y linfoadenopatía mesentérica, nodulaciones en pared intestinal y una severa infección de parásitos blanquecinos y redondos. Al examen coproparasitológico, se evidenciaron huevos característicos del

${ }^{1}$ Laboratorio de Sanidad Animal, Escuela de Medicina Veterinaria, Facultad de Ciencias Agrarias, Universidad Nacional de San Martín - Tarapoto, Tarapoto, Perú

${ }^{2}$ Laboratorio de Histopatología, Escuela de Medicina Veterinaria, Facultad de Ciencias Agrarias, Universidad Nacional de San Martín- Tarapoto, Tarapoto, Perú

${ }^{3}$ Urku, Estudios Amazónicos, Tarapoto, Perú

${ }^{4}$ E-mail: alicialopezflores@unsm.edu.pe; https://orcid.org/0000-0002-4679-6353

${ }^{5}$ E-mail: vhpuicon@unsm.edu.pe; https://orcid.org/0000-0003-2532-2551

Recibido: 24 de julio de 2020

Aceptado para publicación: 27 de marzo de 2021

Publicado: 23 de junio de 2021

CLos autores. Este artículo es publicado por la Rev Inv Vet Perú de la Facultad de Medicina Veterinaria, Universidad Nacional Mayor de San Marcos. Este es un artículo de acceso abierto, distribuido bajo los términos de la licencia Creative Commons Atribución 4.0 Internacional (CC BY 4.0) [https:// creativecommons.org/licenses/by/4.0/deed.es] que permite el uso, distribución y reproducción en cualquier medio, siempre que la obra original sea debidamente citada de su fuente original 
género Prosthenorchis y al análisis del parásito adulto se observaron las ganchos craneales y estructuras características de la especie Prosthenorchis elegans, parásito encontrado en primates neotropicales. Se reporta este caso, no como un hallazgo accidental de necropsia, sino cuya causa de muerte fue a consecuencia de un shock séptico, consecuente de la peritonitis generalizada causado por una severa infección parasitaria de este acantocéfalo que generó una perforación a nivel intestinal.

Palabras clave: Prosthenorchis, acantocéfalo, mono tocón, parásitos

\section{Abstract}

It is reported the case of an adult titi monkey (Plecturocebus oenanthe) parasitized with Prosthenorchis elegans, which died at the end of December 2019. The animal was in a wildlife rescue centre located on the banks of the Shilcayo Quebrada, the entrance gate of the national park «Cordillera Escalera», in San Martín, Peru. The animal, living in semicaptivity, did not show clinical signs, but it collapsed from a height of $5 \mathrm{~m}$. At necropsy, abdominal exudate, perforation at the level of the small intestine, congestion of blood vessels and mesenteric lymphadenopathy, nodulations in the intestinal wall and a severe infection of white and round parasites were evidenced. The coproparasitological examination revealed characteristic eggs of the genus Prosthenorchis and the analysis of the adult parasite revealed the cranial hooks and characteristic structures of the species Prosthenorchis elegans, a parasite found in neotropical primates. This case is reported not as an accidental necropsy finding, but one which cause of death was as a consequence of septic shock due to a generalized peritonitis caused by a severe parasitic infection of this Acantocephalus that generated an intestinal perforation.

Key words: Prosthenorchis, Acanthocephalus, titi, parasites

\section{INTRODUCCIÓN}

Los acantocephala en la fase de huevo de vida libre (acantor) requiere un huésped intermediario de artrópodos para que desarrolle la larva en etapas de acantela y cistacanto, en tanto que el adulto utiliza un huésped vertebrado (Machado Filho, 1950). Las termitas (Nasutitermes acajutlae) han sido identificadas como huésped intermediario y al mono verde africano (Chlorocebus sabaeus) como huésped paraténico (Becker et al., 2019), así mismo, el caso de las cucarachas Blattella germanica con la variedad de hospederos primates como Eulemur macaco (L., 1766), Saguinus oedipus (L., 1758), Saguinus fuscicollis (Spix, 1823), Saguinus midas (L., 1758) (Sokolov et al.,
2016). Estos parásitos representan una preocupación en especies en peligro de extinción (Puttker et al., 2008). La infección por parte de este tipo de parásitos gastrointestinales conlleva a una alta morbilidad y mortalidad, ocasionando daños gastroentéricos que generan desbalances electrolíticos en el animal, con la consecuente pérdida de condición corporal (Pérez et al., 2007).

Prosthenorchis elegans es un parásito intestinal Acanthocephalo reportado en primates titíes de Geoffroy, siendo una amenaza para la supervivencia de este animal en la vida silvestre en el bosque atlántico brasileño (Oliveira et al., 2017). Se ha reportado que la infestación por este parásito está influenciada por la dieta de ingestión de invertebrados de huéspedes intermediarios 
artrópodos como cucarachas, grillos, que son parte de la naturaleza de sus alimentos (Stunkard, 1965; Catenacci et al., 2016). Este acantocéfalo se localiza a nivel de la región ileocecal, generando lesiones como enteritis ulcerosa severa (Campello, 2017), la cual puede conllevar a una peritonitis fulminante causada por la perforación de la pared intestinal del huésped por el parásito (Pissinati et al., 2007). Se reporta que el tratamiento alternativo contra Acanthocephalos (Prosthenorchis elegans) en monos ardilla en cautiverio (Saimiri sciureus) en México consistió en la administración oral de clorhidrato de loperamida $(0.5 \mathrm{mg} / 0.9 \mathrm{~kg})$ y niclosamida $(0.2 \mathrm{mg} / 0.9 \mathrm{~kg})$ durante tres días, seguido de la eliminación quirúrgica de los gusanos adultos del intestino (Zárate et al., 2018).

El Perú cuenta con una alta diversidad de especies de primates en peligro crítico y endémico por su hábitat amenazado (Shanee S y Shanee N, 2018), habiéndose reportado este acantocéfalo en Iquitos (Loreto) en los siguientes primates neotropicales en cautiverio: Aotus azarae, Aotus nancymaae, Ateles panicus, Callicebus cupreus, Cebuella pygmaea, Lagothrix lagotricha, Saguinus fuscicollis, Saguinus labiatus, Saguinus mystax, Saguinus nigricollis, Saimiri boliviensis y Saimiri sciureus (Cárdenas et al., 2010). Este problema sanitario afecta en muchas ocasiones a individuos de especies amenazadas que son albergadas por proyectos de rescate animal con intenciones de repoblamiento en los hábitats originales, donde se presume cierto impacto de este parásito sobre la conservación de dichas especies (Oliveira et al. 2017; Püttker et al., 2008). Es por eso, que es necesario desarrollar métodos que mejoren la precisión del diagnóstico de este tipo de parásitos en muestras no invasivas, y la incorporación de técnicas moleculares en los procedimientos de estudios parasitológicos en primates neotropicales (Solórzano y Pérez, 2018).

En el presente reporte de caso se menciona los signos y daños causados por Prosthenorchis cf. elegans en un ejemplar semi-cautivo de Plecturocebus oenanthe (mono tocón), primate endémico del bosque seco del Huallaga (Región de San Martín) (Shanee S y Shanee N, 2018); considerado en riesgo crítico de extinción por la norma nacional (SERFOR, 2018) y la Lista Roja de la IUCN (Veiga et al., 2011).

\section{Presentación del Caso}

En diciembre de 2019, en el Centro Urku, especializado en la conservación, rescate, rehabilitación y reinserción de especies de fauna silvestre a su medio natural, ubicado en la cuenca media de la quebrada Shilcayo, se reportó que un mono tocón (Plecturocebus oenanthe) adulto, macho, el cual había sido liberado al bosque del área de conservación de Coordillera Escalera, pero que usualmente vivía libremente en este centro, se le halló muerto ante la caída desde una altura de $5 \mathrm{~m}$, caída que fue observada por los encargados y trabajadores del lugar.

A la necropsia realizada a una hora del deceso, se observó ictericia generalizada a nivel de piel y de tejido subcutáneo. En el esófago se encontró un parasito redondo de aproximadamente $3 \mathrm{~cm}$ de largo, sugiriéndose el estadio juvenil del parásito Prosthenorchis elegans (sitio errático). A nivel del aparato cardio-respiratorio se evidenció hipertrofia ventricular concéntrica derecha con congestión de vasos coronarios; así mismo, se evidenció enfisema y leve congestión pulmonar.

En la cavidad abdominal, se evidenció exudado (Figura 1a) y presencia parasitaria. A nivel del estómago, se encontró hemorragia gástrica con mayor intensidad en región antropilórica. A nivel del intestino delgado se observó una severa enteritis difusa y la región terminal del duodeno y del yeyuno presentaba una zona con solución de continuidad, cuyos bordes estaban hemorrágicos y necróticos (Figura 1b). Esta perforación se encontraba asociada a una masiva infección parasitaria sugerente de acantocefaliasis, 

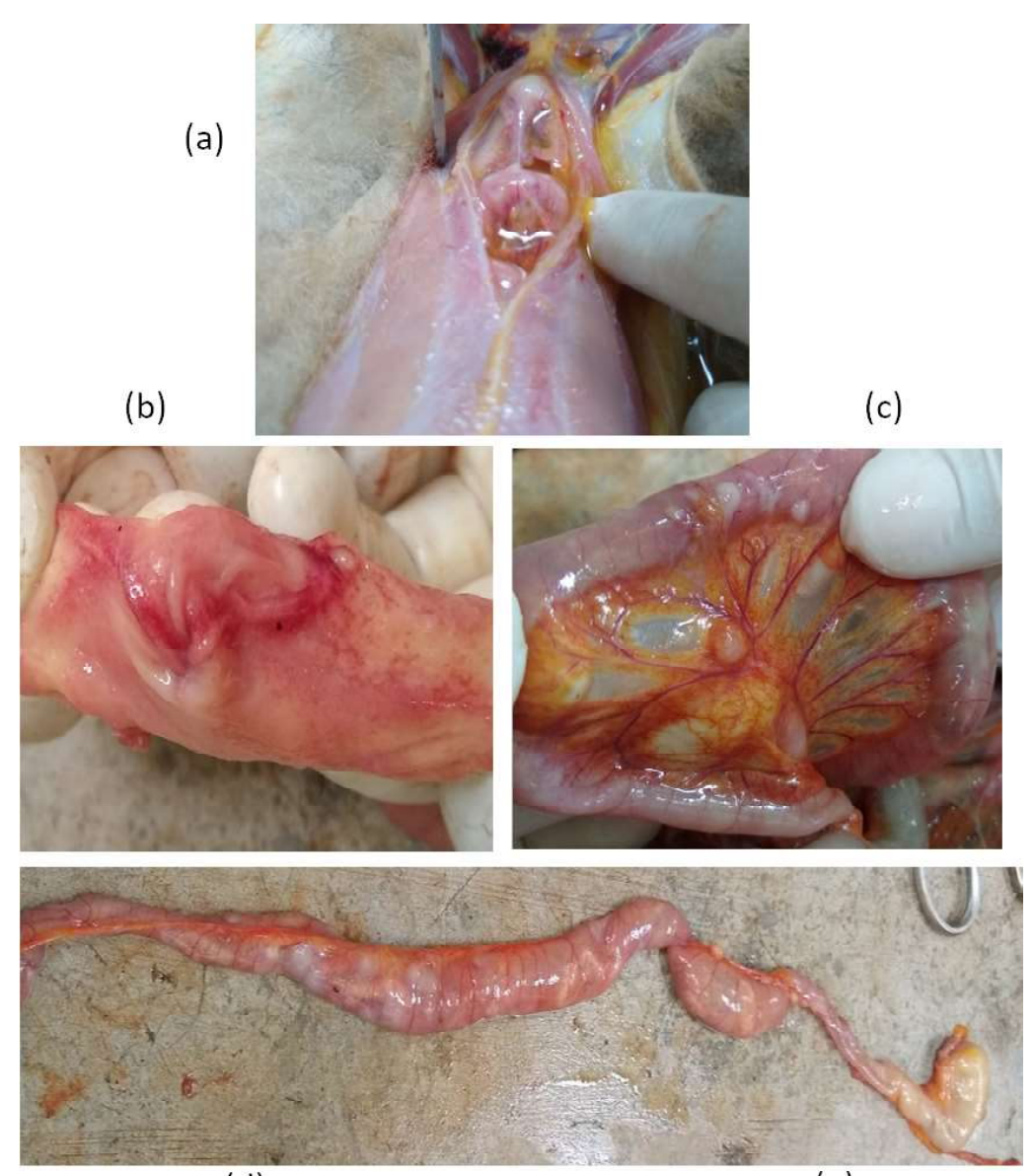

(d)

(e)

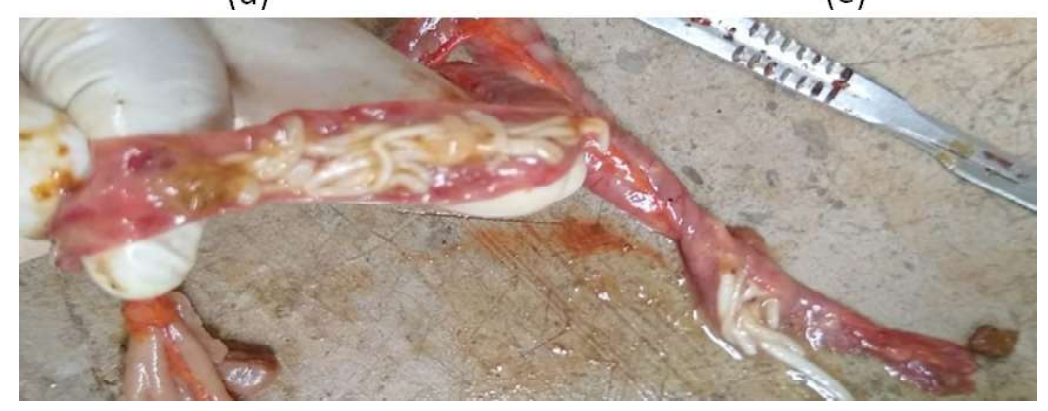

Figura 1. Lesiones macroscópicas halladas en el examen post mortem de un mono tocón (Plecturocebus oenanthe) adulto. (a) Efusión abdominal a la apertura de la cavidad; (b) Solución de continuidad a nivel intestinal, asociado a la adherencia de Prosthenorchis elegans; (c) congestión de vasos mesentéricos y linfoadenopatía regional mesentérica; (d) Nódulos a nivel de la pared intestinal; (e) Apertura de las asas intestinales evidenciando una severa infestación de parásitos blanquecinos y redondos

puesto que en otras zonas intestinales se hallaron los parásitos adheridos fuertemente a la mucosa gastrointestinal; además, se evidenció congestión de los vasos mesentéricos y linfoadenopatía mesentérica (Figura 1c).
Los intestinos presentaban aparentes nódulos (Figura 1d), que evidenciaron una severa infestación de parásitos a su apertura (Figura 1e). Otras lesiones macroscópicas halladas fueron congestión hepática, esplénica, renal, 


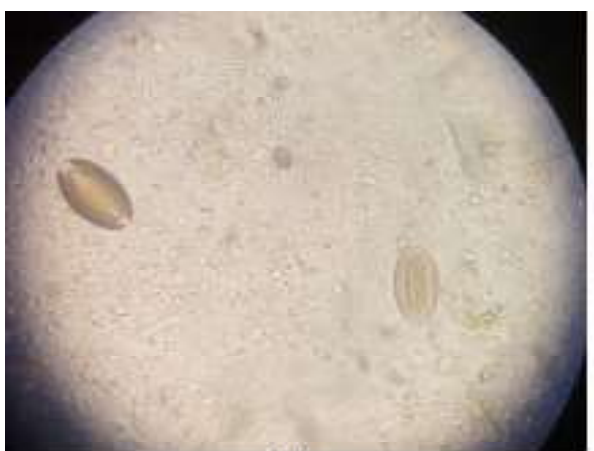

(a)

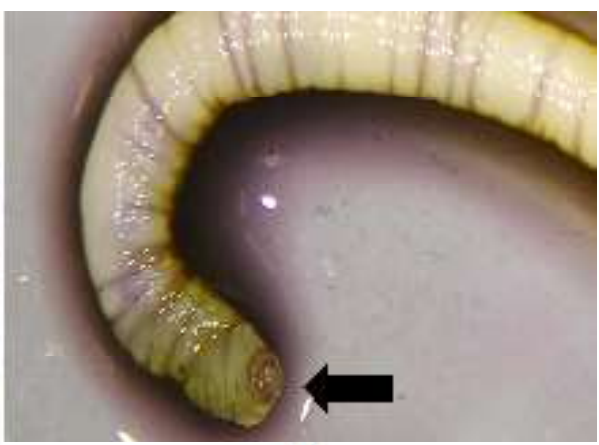

(b)

Figura 2. (a) Huevos de Prosthenorchis elegans mediante el examen coproparasitológico (40X): (b) Forma adulta del parásito. La flecha indica la probóscide, la cual contiene ganchos en su extremo anterior

vesical, cerebral y linfoadenopatía regional mesentérica.

\section{Diagnóstico Parasitológico}

Se evaluaron muestras coprológicas en el Laboratorio de Sanidad Animal de la Escuela Profesional de Medicina Veterinaria, Universidad Nacional de San Martín en Tarapoto, mediante el análisis cualitativo de flotación con solución de $\mathrm{NaCl}$. Se hallaron huevos característicos de Prosthenorchis sp, los cuales se caracterizan por ser ovalados y no poseen opérculo, además de tener tres envoltorios, siendo el externo de superficie rugosa (Figura 2a) (Machado Filho, 1950). Así mismo, en la evaluación estereoscópica del parasito adulto se evidenció de manera característica una serie de pliegues a nivel de todo el tronco con mayor pronunciación en la zona medio anterior, mientras que la cabeza se unía al cuerpo por un collar formado por pliegues del revestimiento externo, con un aspecto característico, el cual estaba conformado por fuertes ganchos, en número de seis (Figura 2b). Las características del hue- vo y del parasito adulto están acorde a lo reportado por Machado Filho (1950), por lo que ambos análisis confirmaron el diagnóstico de acantocefaliasis causado por Prosthenorchis elegans.

\section{Diagnóstico Histopatológico}

Se tomaron muestras de intestino delgado, nódulo linfático mesentérico, pulmón y riñón. Se realizó el examen microscópico en el Laboratorio de Histopatología Animal de la Escuela Profesional de Medicina Veterinaria, Universidad Nacional de San Martín en Tarapoto. Anivel histopatológico se evidencia un gran contenido amorfo y acidofília intensa compatible con necrosis caseosa con proyecciones capsulares fibrosas, depositado a nivel de submucosa y túnica muscular intestinal ( $\mathrm{Fi}$ guras 3a,b,c); en las fibras musculares se observaron artefactos denominados «Venecian blints». Asimismo, se observó nodo linfático reactivo (Figura 4a), con predominio de una población mononuclear (Figura 4b) y leve congestión pulmonar con presencia de enfisema y colapso alveolar (Figura 4c). 
(a)

(b)

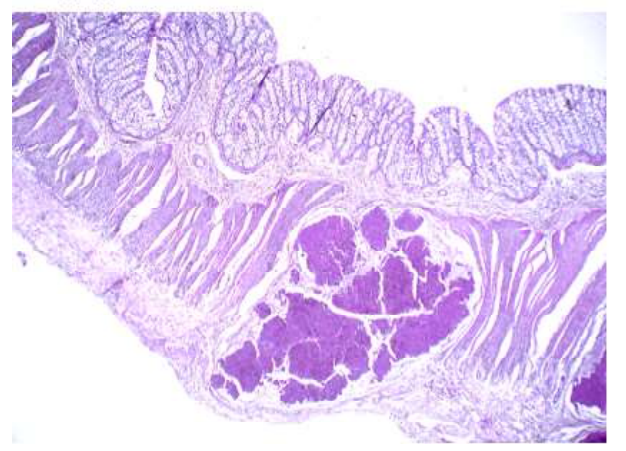

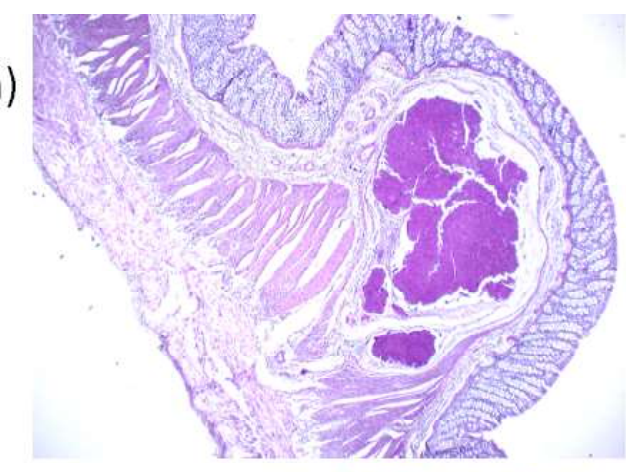

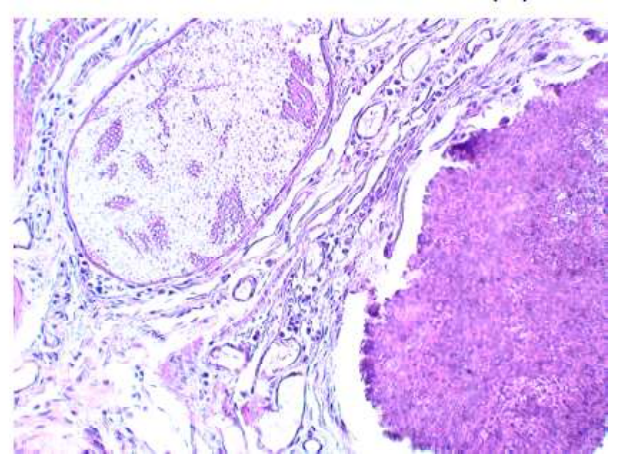

Figura 3. Lesiones histopatológicas en un mono tocón (Plecturocebus oenanthe) adulto. (a) Necrosis caseosa a nivel de la submucosa intestinal, (b) Necrosis caseosa a nivel de la túnica muscular y serosa, se distingue los artefactos «Venecian blints» a nivel de la túnica muscular, (c) capa de fibroblastos y mononucleares rodeando a la necrosis caseosa

(a)

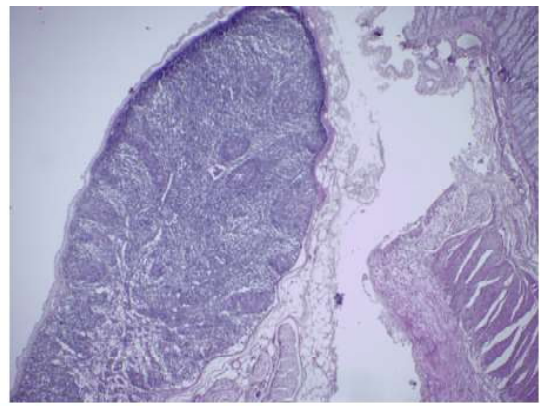

(c)

(b)

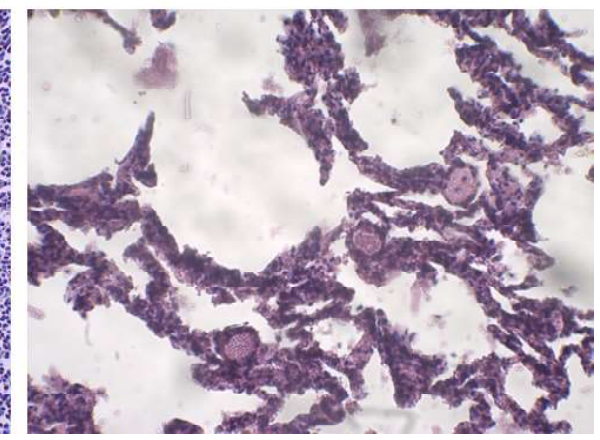

Figura 4. Lesiones histopatológicas en un mono tocón (Plecturocebus oenanthe) adulto. (a) Nodo linfático reactivo, (b) Población mononuclear de nodo linfático reactivo, (c) Congestión pulmonar, enfisema y colapso alveolar 


\section{Discusión}

E1 mono tocón (Plecturocebus oenanthe) es una de las especies que se encuentran en situación de riesgo crítico. En la actualidad no se encuentra en situación de protección dentro de las unidades de conservación y protección gubernamental de la Región San Martín, y su hábitat natural se encuentra en reducción constante por la acelerada deforestación, particularmente en zonas como el Alto Mayo y Huallaga Central (IIAP, 2006).

La literatura menciona parásitos como protozorios y helmintos en una variedad de primates no humanos albergados en reservas naturales (Phillips et al., 2004; Oliveira et al., 2017) y en centros de cautiverio (Martin-Solano et al., 2017); reportándose a los acantocéfalos como causantes de casos de mortalidad (Catenacci et al., 2016; Oliveira et al., 2017; Zárate et al., 2018) y con mal pronóstico, incluso luego de tratamientos quirúrgicos (Rojas et al., 2016).

En relación a los acantocéfalos, también denominados gusanos de cabeza espinosa, se han reportado tres especies que parasitan a los primates no humanos: Prosthenorchis elegans, Prosthenorchis spirula y Moniliformis clarki (Dunn, 1963). P. elegans es un acantocéfalo, cuyos huevos son coprológicamente indistinguibles en el diagnóstico diferencial con respecto a $P$. spirula (Baker, 2008; Falla et al., 2015); sin embargo, presenta características morfológicas adultas que lo diferencia, puesto que en la última especie, se denota rugosidades más acentuadas en la parte mediana y carece de segmentación, siendo la parte anterior, más dilatada y el doble de la extremidad posterior (Machado Filho, 1950), por lo que se le pudo diferenciar al observar especímenes obtenidos durante la necropsia.

Los parásitos adultos se localizan a nivel del lumen del íleo terminal, ciego y colon, que con sus ganchos craneales adquieren una adhesión fija dentro de la submucosa y la capa muscular intestinal, con riesgo de debridamiento tisular, consecuente perforación y complicaciones como peritonitis y muerte del animal (Dunn, 1963; Parr et al., 2013; Oliveira et al., 2017). En el caso reportado, los parásitos se hallaron libres y adheridos a nivel de la mucosa gastrointestinal, más aún en zonas como en la porción final del duodeno y yeyuno, a diferencia con lo reportado en la literatura.

El mono tocón se encontraba en los alrededores del centro de rescate, luego de haber pasado una temporada en cautiverio; sin embargo, el animal tenía la predilección de merodear y permanecer dentro de las instalaciones del centro. En los días previos a su muerte, el animal se mantenía activo, el apetito no se había alterado y constantemente paseaba de un lugar a otro, sin presentar signos clínicos aparentes como depresión, hipotermia, emaciación, los cuales han sido reportados previo a la muerte de animales parasitados con Prosthenorchis sp (Rojas et al., 2016). Sin embargo, en el día de su muerte solo se le observó escondido en un árbol, tiempo en el cual, el animal pudo haber desarrollado un shock septicémico como hipotensión arterial, fiebre, hipoxia y alteración del sensorio (McGavin y Zachary, 2007).

Los hallazgos de la necropsia como ictericia cutánea y subcutánea pueden explicarse por la obstrucción y bloqueo de los conductos biliares a nivel del duodeno, los cuales fueron evidenciados en referencia a la localización de los parásitos en la necropsia. Además, la evidencia de la adherencia del extremo anterior de los parásitos a la mucosa gastrointestinal sugiere que las perforaciones a nivel del intestino sean causadas por estos, causando una severa enteritis necrótica multifocal crónica. De otra parte, se halló exudado abdominal con presencia de contenido alimenticio y de parásitos a nivel de la cavidad abdominal, con lo cual se generó una peritonitis generalizada, con proliferación bacteriana y liberación de toxinas conduciendo a un shock, con lo cual produjo una insuficiencia respiratoria causando la muerte del 
animal (Catenacci et al., 2016; Oliveira et al., 2017).

Se reporta este caso, no como un hallazgo accidental de necropsia, sino cuya causa de muerte fue a consecuencia de un shock séptico, consecuente de la peritonitis generalizada causado por una severa infección parasitaria de este acantocéfalo que generó una perforación a nivel intestinal. Cabe considerar que la epidemiologia de la prostenorquiosis se basa en la distribución de los hospederos intermediarios, las cucarachas (Blatella germanica), las cuales conforman como parte de la dieta de estos animales (Pissinatti et al., 2007) o cuando los primates no humanos habitan en ambientes cercanos al humano (Wenz et al., 2010).

\section{Literatura Citada}

1. Baker DG 2008. Flynn's parasites of laboratory animals. $2^{\circ}$ ed. Oxford: Blackwell Publishing. $840 \mathrm{p}$.

2. Becker AAMJ, Rajeev S, Freeman MA, Beierschmitt A, Savinon V, Wulcan JM, Bolfa P. 2019. Extraintestinal Acanthocephalan Oncicola venezuelensis (Oligacanthorhynchidae) in small Indian mongooses (Herpestes auropunctatus) and African green monkeys (Chlorocebus aethiops sabaeus). Vet Pathol 6: 794-798. doi: 10.1177/0300985819848502

3. Campello L. 2017. Relato de caso: Prosthenorchis elegans (parasita acantocéfalo) em Saimiri sp (macacode-cheiro) em centro de primatologia brasileiro. Tese de Médico Veterinário . Brasilia, Brasil. $50 \mathrm{p}$.

4. Cárdenas JM. 2010. Presencia de Prosthenorchis elegans (Diesing, 1851) en primates neotropicales obtenidos por caza de subsistencia en la comunidad nueva esperanza, Loreto - Perú. En: II Congreso Internacional de Parasitología Neotropical (II COPANEO). Lima, Perú.
5. Catenacci LS, Pessoa MS, NogueiraFilho SL De Vleeschouwer K. 2016. Diet and feeding behavior of Leontopithecus chrysomelas (Callitrichidae) in degraded areas of the Atlantic forest of South-Bahia, Brazil. Int J Primatol 37: 136157. Doi: 10.1007/s10764-016-9889-x

6. Dunn FL. 1963. Acanthocephalans and cestodes of South American monkeys and marmosets. J Parasitol 49: 717e722.

7. Falla A, Brieva C, Bloor P. 2015. Mitochondrial DNA diversity in the acanthocephalan Prosthenorchis elegans in Colombia based on cytochrome c oxidase I (COI) gene sequence. Int J Parasitol 4: 401-407. doi: 10.1016/ j.ijppaw.2015.08.002

8. [IIAP] Instituto de Investigaciones de la Amazonia Peruana. 2006. Estrategia regional de la diversidad biológica de San Martín. San Martín, Perú: Comisión Regional de San Martín. Informe Técnico. $119 \mathrm{p}$.

9. Machado-Filho DA. 1950. Revisão do gênero Prosthenorchis Travassos, 1915 (Acanthocephala). Mem I Oswaldo Cruz 48: 495-544. doi: 10.1590/S007402761950000100020

10. Martin-Solano S, Carrillo-Bilbao GA, Ramirez W, Celi-Erazo M, Huynen MC, Levecke B, Benitez-Ortiz W, Losson B. 2017. Gastrointestinal parasites in captive and free-ranging Cebus albifrons in the Western Amazon, Ecuador. Int J Parasitol Parasites Wildl 6: 209-218. doi: 10.1016/j.ijppaw.2017.06.004

11. McGavin MD, Zachary JF. 2007. Pathologic basis of veterinary disease. $4^{\text {th }}$ ed. Missouri: Mosby Elsevier. $1476 \mathrm{p}$.

12. Oliveira AR, Hiura E, Guião-Leite FL, Flecher MC, Braga FR, Silva LP, Sena T, Souza TD. 2017. Pathological and parasitological characterization of Prosthenorchis elegans in a freeranging marmoset Callithrix geofroyi from the Brazilian Atlantic Forest. Pesqui Vet Brasil 37: 1514-1518. doi: 10.1590/ s0100-736x2017001200025 
13. Parr NA, Fedigan LM, Kutz SJ. 2013. A coprological survey of parasites in white faced capuchins (Cebus capucinus) from Sector Santa Rosa, ACG, Costa Rica. Folia Primatol 84: 102-114. doi: $10.1159 / 000348287$

14. Pérez JL, Hernández M, Pisos E, Carranza C. 2007. Tratamiento de las enfermedades parasitarias (II): Helmintosis y ectoparasitosis. Inf Ter Sist Nac Salud 31: 55-64.

15. Phillips KA, Haas ME, Grafton BW, Yrivarren M. 2004. Survey of the gastrointestinal parasites of the primate community at Tambopata National Reserve, Peru. J Zool 264: 149-151. doi: 10.1017/S0952836904005680

16. Pissinatti L, Pissinatti A, Burity $\mathrm{CH}$, Mattos DG, Tortelly R. 2007. Ocorrência de Acanthocephala em Leontopithecus (Lesson, 1840), cautivos: aspectos clínico-patológicos. Callitrichidae-Primates. Arq Bras Med Vet Zoo 59: 1473-1477. doi: 10.1590/S010209352007000600019

17. Püttker T, Meyer-Lucht Y, Sommer S. 2008. Effects of fragmentation on parasite burden (nematodes) of generalist and specialist small mammal species in secondary forest fragments of the coastal Atlantic Forest, Brazil. Ecol Res 23:207-215.

18. Rojas ZE, Segura VM, Rincón MT, Granados JL, Brieva C. 2016. Intususcepción ileal generada por Prosthenorchis sp en un tití gris (Saguinus leucopus) mantenido en un centro de recepción de fauna en Colombia. Rev Med Vet 31: 75-83.

19. Shanee S, Shanee N. 2018. Diversity of large mammals in the MarañónHuallaga landscape, Perú: with notes on rare species. Zool Ecol 28: 313-328. doi: 10.1080/21658005.2018.1516277

20. [SERFOR] Servicio Nacional Forestal y de Fauna Silvestre, Ministerio de Agricultura, Perú. 2018. Libro rojo de la fauna silvestre amenazada del Perú. Lima, Perú: Serfor. 558 p.

21. Sokolov SG, Alshinetsky MV, Berezin MV, Efeykin BD, Spiridonov SE. 2016. Acanthocephalans Prosthenorchis cf. elegans (Archiacanthocephala: Oligacanthorhynchidae), parasites of primates in the Moscow zoo. Parazitologiia 50: 185-196.

22. Solórzano B, Pérez G. 2018. Parasites of Neotropical Primates: a review. Int J Primatol 39: 155-182. doi: 10.1007/ s10764-018-0031-0

23. Stunkard HW. 1965. New intermediate hosts in the life cycle of Prosthenorchis elegans (Diesing, 1851), an acanthocephalan parasite of primates. J Parasitol 51: 645-649

24. Veiga L, Bóveda-Penalba A, Vermeer J, Tello-Alvarado JC, Cornejo F. 2011. Plecturocebus oenanthe. The IUCN Red List of Threatened Species 2011. doi: 10.2305/IUCN.UK.2008.RLTS.T41559A10478781.en

25. Wenz A, Heymann EW, Petney TN, Taraschewski HF. 2009. The influence of human settlements on the parasite community in two species of Peruvian tamarin. Parasitology 137: 675-684. doi: $10.1017 / \mathrm{S} 0031182009991570$

26. Zárate JJ, Gómez MA, Rodríguez LE, Hernández J, Contreras JA. 2018. An alternative treatment against acanthocephala (Prosthenorchis elegans) in captive squirrel monkeys (Saimiri sciureus) in Mexico. J Parasitol 104: 574-575. doi: 10.1645/17-93 